Background: COVID-19 can induce a hyperinflammatory state resulting in cytokine storm, which can lead to poor outcomes. Patients with systemic rheumatic diseases may be at increased risk for respiratory failure with COVID-19. Therefore, we investigated the relationship between rheumatic disease, hyperinflammation, and clinical outcomes among hospitalized COVID-19 patients.

Objectives: To compare laboratory values, hyperinflammation, and clinical outcomes of hospitalized COVID-19 rheumatic patients and matched comparators.

Methods: We performed a comparative cohort study of patients with polymerase chain reaction (PCR)-confirmed COVID-19 requiring hospitalization between $3 / 1 / 20-7 / 7 / 20$ at a large health care system. We compared each systemic rheumatic disease case to up to 5 matched (by age, sex, and date of +SARS-CoV-2 PCR) comparators without systemic rheumatic disease. We extracted laboratory values from their hospitalization to compare peaks/troughs of individual laboratory results by case status and derived the COVID-19-associated hyperinflammation score (cHIS), a composite of 6 laboratory domains $(0-6, \geq 2$ indicating hyperinflammation), as previously developed ${ }^{1}$. We used multivariable logistic regression to estimate ORs for COVID-19 outcomes by hyperinflammation and case status.

Results: We identified 57 hospitalized rheumatic disease cases (mean age 67 years, 67\% female) and 232 matched comparators hospitalized with PCR-confirmed COVID-19. Among cases, 26 (46\%) had rheumatoid arthritis and 14 $(25 \%)$ had systemic lupus erythematosus. Most cases $(34,60 \%)$ had active rheumatic disease. At baseline, $15(27 \%)$ of cases were treated with biologic DMARDs, and 32 (56\%) were using glucocorticoids. We analyzed 39,900 total laboratory results (median 85 per patient). Cases had higher peak neutrophil-to-lymphocyte ratio ( 9.6 vs $7.8, p=0.02$ ), $\mathrm{LDH}$ (421 vs $345 \mathrm{U} / \mathrm{L}, p=0.04$ ), creatinine (1.2 vs $1.0 \mathrm{mg} / \mathrm{dL}, p=0.01$ ), and BUN (31 vs $23 \mathrm{mg} / \mathrm{dL}, p=0.03$ ) than comparators but similar peak CRP (149 vs $116 \mathrm{mg} / \mathrm{L}, p=0.11$, Figure 1 ). Cases had higher peak median cHIS ( 3 vs $2, p=0.01$ ). Peak $\mathrm{cHIS} \geq 2$ had higher odds of intensive care unit (ICU) admission (OR 3.45, 95\%Cl 1.98-5.99), mechanical ventilation (OR $66.0,95 \% \mathrm{Cl} 9.0-487.8$ ), and mortality (OR $16.4,95 \% \mathrm{Cl}$ 4.8-56.4) compared to cHIS <2 (Table 1). Cases had increased risk of ICU admission (OR 2.0, 95\% Cl 1.1-3.7) and mechanical ventilation (OR 2.7, 95\% Cl 1.4-5.2) than comparators

Table 1. Associations of peak cHIS and systemic rheumatic disease with COVID-19 hospitalization outcomes

\begin{tabular}{|c|c|c|c|c|c|c|}
\hline & \multicolumn{2}{|c|}{$\begin{array}{l}\text { Intensive care unit } \\
\text { admission }\end{array}$} & \multicolumn{2}{|c|}{ Mechanical ventilation } & \multicolumn{2}{|r|}{ Death } \\
\hline & $\%$ & $\begin{array}{l}\text { Adjusted OR } \\
\qquad(95 \% \mathrm{Cl})\end{array}$ & $\%$ & $\begin{array}{l}\text { Adjusted OR } \\
\qquad(95 \% \mathrm{Cl})\end{array}$ & $\%$ & $\begin{array}{l}\text { Adjusted OR } \\
\qquad(95 \% \mathrm{Cl})\end{array}$ \\
\hline \multicolumn{7}{|c|}{ Hospitalization outcomes by hyperinflammation on $\mathrm{cHIS}^{1}$} \\
\hline cHIS <2 (n=112) & $21 \%$ & 1.0 (Ref) & $1 \%$ & 1.0 (Ref) & $3 \%$ & 1.0 (Ref) \\
\hline $\mathrm{cHIS} \geq 2(\mathrm{n}=177)$ & $48 \%$ & $3.5(2.0-6.0)$ & $37 \%$ & $66.2(9.0-487.8)$ & $27 \%$ & $16.4(4.8-56.4)$ \\
\hline \multicolumn{7}{|c|}{ Hospitalization outcomes by rheumatic disease status } \\
\hline $\begin{array}{l}\text { Comparators } \\
\quad(n=232)\end{array}$ & $30 \%$ & 1.0 (Ref) & $19 \%$ & 1.0 (Ref) & $16 \%$ & 1.0 (Ref) \\
\hline $\begin{array}{l}\text { Rheumatic cases } \\
\quad(n=57)\end{array}$ & $51 \%$ & $1.87(1.03-3.40)$ & $39 \%$ & $2.46(1.30-4.67)$ & $21 \%$ & $1.32(0.61-2.88)$ \\
\hline
\end{tabular}

Matching factors: age, sex, and date of $+\mathrm{PCR} .{ }^{1}$ Adjusted for age, sex, and case status. ${ }^{2}$ Adjusted for race, smoking, comorbidities, and body mass index.cHIS, COVID19-associated hyperinflammation score; $\mathrm{Cl}$, confidence interval; OR, odds ratio; PCR polymerase chain reaction; SARS-CoV-2, severe acute respiratory syndrome coronavirus 2 .

Conclusion: Patients with systemic rheumatic disease hospitalized for COVID19 had higher risk for hyperinflammation, kidney injury, and mechanical ventilation than non-rheumatic comparators. We validated the cHIS in our cohort, which was strongly associated with poor COVID-19 outcomes. These findings highlight that hospitalized patients with rheumatic diseases may be vulnerable to poor COVID-19 outcomes.

REFERENCES:

[1] Webb BJ et al. Clinical criteria for COVID-19-associated hyperinflammatory syndrome. Lancet Rheumatol. 2020 Dec;2(12):e754-e763.
Figure:

Median peak laboratory values and composite COVID-19-associated hyperinflammation scores (cHIS) for systemic rheumatic disease cases $(n=57)$ and matched comparators $(n=232)$ during hospitalization for COVID-19. Error bars indicate interquartile range. cHIS was derived from 39,900 laboratory results, and the components are listed.


Disclosure of Interests: Tiffany Hsu: None declared, Kristin D'Silva: None declared, Naomi Serling-Boyd: None declared, Jiaqi Wang: None declared, Alisa Mueller: None declared, Xiaoqing Fu: None declared, Lauren Prisco: None declared, Lily Martin: None declared, Kathleen Vanni: None declared, Alessandra Zaccardelli: None declared, Claire Cook: None declared, Hyon Choi Consultant of: Dr. Choi reports consultancy fees from Takeda, Selecta, GlaxoSmithKline, and Horizon, Grant/research support from: Dr. Choi reports research support from AstraZeneca., Yuqing Zhang: None declared, Ellen Gravallese: None declared, Zachary Wallace Consultant of: Dr. Wallace reports consulting fees from Viela Bio and MedPace., Grant/research support from: Dr. Wallace reports research support from Bristol-Myers Squibb and Principia., Jeffrey Sparks Consultant of: Dr. Sparks reports consultancy fees from Bristol-Myers Squibb, Gilead, Inova, Janssen, Optum, and Pfizer., Grant/ research support from: Dr. Sparks reports research support from Amgen and Bristol-Myers Squibb.

DOI: 10.1136/annrheumdis-2021-eular.936

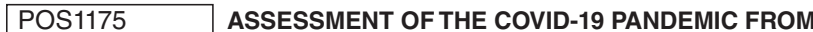 THE PERSPECTIVE OF PEOPLE WITH RHEUMATIC MUSCULOSKELETAL DISEASES IN EUROPE. RESULTS FROM THE REUMAVID STUDY (PHASE 1)}

M. Garrido-Cumbrera ${ }^{1}$, H. Marzo-Ortega ${ }^{2}$, J. Correa-Fernández ${ }^{1}$, S. SanzGómez ${ }^{1}$, L. Christen ${ }^{3}$, V. Navarro-Compán ${ }^{4}$ on behalf of REUMAVID working group. ${ }^{1}$ Universidad de Sevilla, Health \& Territory Research (HTR), Sevilla, Spain; ${ }^{2}$ University of Leeds, Leeds Institute for Rheumatic and Musculoskeletal Medicine, Leeds, United Kingdom; ${ }^{3}$ Novartis Pharma AG, Patient Engagement, Basel, Switzerland; ${ }^{4}$ Hospital Universitario La Paz, IdiPaz, Madrid, Spain

Background: The COVID-19 pandemic is an unprecedented public health crisis affecting people worldwide, including those with rheumatic and musculoskeletal diseases (RMDs). 
Objectives: REUMAVID aims to assess the impact of the COVID-19 pandemic and lockdown on the wellbeing, mental health, disease activity and function, access to health care and treatment, support services, and hopes and fears of people RMDs.

Methods: REUMAVID is an international collaboration led by the Health \& Territory Research group at University of Seville, Spain, together with a multidisciplinary team including patient organization and rheumatologists. This cross-sectional study consisting of an online survey gathering data from patients with a diagnosis of 15 RMDs in Cyprus, France, Greece, Italy, Portugal, Spain and the United Kingdom. Participants are recruited by patient organizations. Data is collected in two phases: 1) during the first peak of the COVID-19 pandemic (Spring 2020), and 2) as a follow-up to the pandemic (Winter 2020). This analysis presents descriptive results of the aggregated data, summarizing continuous and categorical variables

Results: A total of 1,800 RMD patients have participated in the first wave of the COVID-19 pandemic (from early April to mid-June 2020). The most frequent reported diagnosis were axial spondyloarthritis (37.2\%), rheumatoid arthritis $(29.2 \%)$ and osteoarthritis (17.2\%). Mean age was $52.6 \pm 13.2,80.1 \%$ were female, $69.6 \%$ were in a relationship or married and $48.6 \%$ had university studies. In total, $1.1 \%$ had tested positive for COVID-19, $10.8 \%$ reported symptoms but were not tested, while $88.1 \%$ did not experience any symptoms. $46.6 \%$ reported worsening health during the pandemic. $63.9 \%$ perceived their health status to be "fair to very bad". Access to care was limited with $58.4 \%$ being unable to keep the rheumatologist appointment, of which, $35.2 \%$ were cancelled by the provider and $54.4 \%$ was attended by phone or online. $15.8 \%$ changed their medication, for which $65.5 \%$ were changed by the provider and $24.6 \%$ by own decision. Reported wellbeing and psychological health during the pandemic was poor, with $49.0 \%$ reporting poor wellbeing according to the WHO-5 scale, $57.3 \%$ marking as anxiety and $45.8 \%$ as depression in the HADS scale. During the pandemic, $24.6 \%$ smoked and $18.2 \%$ drank more than before and $54.5 \%$ were unable to exercise at home.

Conclusion: Results from the first phase of REUMAVID show disturbance of the healthcare quality, substantial changes in harmful health behaviors and an unprecedented impairment of mental health in REUMAVID participants. REUMAVID will continue to collect information in order to assess the impact of the COVID-19 pandemic in people affected by RMDs across Europe.

Acknowledgements: This study was supported by Novartis Pharma AG. We would like to thank all patients that completed the survey as well as all of the patient organisations that participated in the REUMAVID study including: the Cyprus League Against Rheumatism (CYPLAR) from Cyprus, the Association Française de Lutte Anti-Rhumatismale (AFLAR) from France, the Hellenic League Against Rheumatism (ELEANA) from Greece, the Associazione Nazionale Persone con Malattie Reumatologiche e Rare (APMARR) from Italy, the Portuguese League Against Rheumatic Diseases (LPCDR), from Portugal, the Spanish Federation of Spondyloarthritis Associations, the Spanish Patients' Forum (FEP), UNiMiD, Spanish Rheumatology League (LIRE), Andalusian Rheumatology League (LIRA), Catalonia Rheumatology League and Galician Rheumatology League from Spain, and the National Axial Spondyloarthritis Society (NASS), National Rheumatoid Arthritis (NRAS) and Arthritis Action from the United Kingdom.

Disclosure of Interests: Marco Garrido-Cumbrera: None declared, Helena Marzo-Ortega Speakers bureau: AbbVie, Biogen, Celgene, Janssen, Lilly, Novartis, Pfizer, Takeda and UCB, Consultant of: AbbVie, Celgene, Janssen, Lilly, Novartis, Pfizer and UCB, Grant/research support from: Janssen and Novartis, José Correa-Fernández: None declared, Sergio Sanz-Gómez: None declared, Laura Christen Employee of: Novartis Pharma AG, Victoria Navarro-Compán Grant/ research support from: Abbvie, BMS, Janssen, Lilly, MSD, Novartis, Pfizer, Roche, and UCB.

DOI: 10.1136/annrheumdis-2021-eular.956

\section{POS1176 THE IMPACT OF COVID-19 QUARANTINE ON MENTAL HEALTH OF PATIENTS WITH FIBROMYALGIA}

F. Ingegnoli ${ }^{1}$, M. Buoli ${ }^{2}$, C. Posio ${ }^{3}$, R. DI Taranto ${ }^{1}$, R. Caporali ${ }^{1}{ }^{1}$ Università degli Studi di Milano, Division of Clinical Rheumatology, ASST Pini, Department of Clinical Sciences \& Community Health, Research Center for Adult and Pediatric Rheumatic Diseases, Milano, Italy; ${ }^{2}$ Università degli Studi di Milano, Department of Pathophysiology and Transplantation, Department of Neurosciences and Mental Health, Fondazione IRCCS Ca'Granda Ospedale Maggiore Policlinico, Milano, Italy, Milano, Italy; ${ }^{3}$ Università degli Studi di Milano, Department of Pathophysiology and Transplantation, Milano, Italy

Background: Detrimental psychological effects, including symptoms of post-traumatic stress disorder (PTSD), stress, anxiety, and depression have been related to COVID-19 quarantine measures (1). These aspects may be particularly relevant in stress-related disorders such as fibromyalgia (FM) in which previous evidence demonstrated a causal effect of psychological stressors on chronic non-inflammatory pain of FM (2). Recent studies highlighted a significant worsening of FM symptoms after COVID-19 related lockdown period (3-4), but the levels of perceived stress and distress have not been evaluated yet.

Objectives: The aim of this study is to establish the COVID-19 related perceived stress and distress among patients with FM who experienced the COVID-19 quarantine in Italy. Data were retrieved from a larger nationwide online survey involving patients affected by different rheumatic diseases (RD).

Methods: We collected data from May to September 2020 from RD patients living in Italy during the COVID-19 quarantine by an ad-hoc online survey. Eleven associations of RD patients sent a call by using their mailing list, webpages and social network. They asked to complete an anonymous online survey which included the Perceived Stress Scale (PSS), and the Impact Event Scale-Revised (IES-R). Descriptive statistics were used to summarize results.

Results: 79 of 507 (15.6\%) of RD patients who completed the survey declared to have been diagnosed FM. $77(97.5 \%)$ were females, with a mean ( \pm SD) age of $51.0 \pm 12.4 \mathrm{yrs}$. In FM patients, the mean (SD) PSS score was $23.8 \pm 8.1$. In particular, $8(10.1 \%)$ reported low, $38(48.1 \%)$ moderate and $33(41.8 \%)$ high PSS scores. Moreover, IES-R mean (SD) score was $38.5 \pm 17.2$. Among FM patients, $51(64.6 \%)$ reported scores greater than 33 , indicating the probable presence of a PTSD. With regard to the IES-R subscale scores, the total sample did not show a prominence of one of the three main domains: intrusion (13.9 $7.3)$, avoidance $(11.9 \pm 6.1)$ and hyperarousal $(12.6 \pm 5.9)$. In these FM patients, self-reported major sources of anxiety were related to personal and relatives health $(59.5 \%)$, followed by social withdrawal $(21.5 \%)$ and employment loss/ financial difficulties (11.4\%). There was a slight increase of self-reported use of antidepressants (15 [18.9\%] vs 16 [20.2\%]) and anxiolytics drugs (4 [5.1\%] vs 5 [6.3\%]) after lockdown period.

Conclusion: These results confirm that psychosocial stress and distress were highly pervasive in FM after COVID-19 national lockdown. It is known that stressed status may exacerbate and/or precipitate later development of FM symptoms. These data support the substantial worsening of somatic burden of FM after lockdown period reported in previous studies (3-4). Coping strategies should be implemented to ameliorate psychological well-being of these patients in this stressful era.

\section{REFERENCES:}

[1] Brooks SK, et al. Lancet. 2020;395:912-20.

[2] Hung $\mathrm{CH}$, et al. Ann Rheum Dis 2020;79:1644-1656.

[3] Batres-Marroquín AB, et al. J Clin Rheumatol. 2020 Epub ahead of print.

[4] Cavalli G, et al. Rheumatology 2021;60:465-467.

Acknowledgements: We wish to thank the Lombard Association of Rheumatic Diseases (ALOMAR) for its invaluable contribution to the planning and dissemination the survey, all the Italian associations among which the National Association of People with Rheumatic and Rare Diseases (APMARR) and National Association of People with Rheumatic Diseases (ANMAR) that disseminated the survey through social media. The authors are grateful to all patients for contributing to this project.

Disclosure of Interests: Francesca Ingegnoli: None declared, Massimiliano Buoli: None declared, Cristina Posio: None declared, Raffaele Di Taranto: None declared, Roberto Caporali Speakers bureau: Abbvie, Amgen, BMS, Celltrion, Galapagos, Gilead, Lilly, Pfizer, Roche, UCB, Sanofi, Fresenius Kabi, Samsung bioepis, MSD, Consultant of: Galapagos, Gilead, Lilly,Janssen, MSD. DOI: 10.1136/annrheumdis-2021-eular.984

\section{POS1177 REVAMPING BIOLOGIC THERAPY DURING COVID-19}

S. Ahmed Narikkoottungal ${ }^{1}$, A. Siddiqui ${ }^{1}$, A. Constantin ${ }^{1}$, S. Farrow ${ }^{1}$, K. Ahmed ${ }^{1}$. ${ }^{1}$ Princess Alexandra Hospital, Department of Rheumatology, Harlow, United Kingdom

Background: The COVID-19 pandemic has caught us all by surprise - from governments to individuals; the medical fraternity being no exception. It has affected all walks of life; with its immense contagiosity, diverse and intriguing pathogenesis and manifestations differing from other viruses. It has indeed left humanity in dark, unchartered waters; particularly in the early months of the pandemic. Objectives: This article shares the experience, in a Rheumatology department in a District General Hospital (DGH) in the United Kingdom, of managing patients on Biologic (b) and Targeted Synthetic (ts) DMARDs, in the midst of the COVID19 Pandemic.

Methods: All Rheumatology patients at the Princess Alexandra Hospital (PAH) in Harlow newly started on a biologic or targeted synthetic DMARD between 3rd July and 3rd Oct 2020 were identified. These patients had active inflammatory arthritis. Each patient was discussed in a dedicated Multi-Disciplinary Team (MDT) meeting and a consensus on treatment reached in-line with local and National guidelines. 